\title{
CARE OF THE PATIENT WITH FRACTURE NECK OF FEMUR FOR NON-EMERGENCY SURGERY
}

\author{
Dr K Balakrishnan \\ Honorary Consultant Anaesthetist at Southern Railway Hospital, Chennai, India \\ *Corresponding author : balakrishnan.kanni@gmail.com
}

Key words: fracture, neck of femur

Hip fracture becomes one of the most common surgical procedures for patients after the $6^{\text {th }}$ decade of life. The frequency of this occurrence will increase as the percentage of advanced-age population increases, reflecting the progressive aging of the population going into the twentyfirst century. The financial and social impact of a hip fracture is tremendous, permanently altering the lives of a high percentage of those affected. One of the biggest challenges that face the anaesthesiologists while offering perioperative care for these patients is the concurrent coexisting diseases and the decreased organ reserves. However, with advancements in our understanding of the pathophysiology of ageing, pharmacotherapy, safer anaesthetic techniques, advanced monitoring, better surgical solutions, multimodal pain therapy, early mobilization protocols, proactive physiotherapy, counseling and rehabilitation, the perioperative morbidity and mortality has considerably decreased

\section{Aetiology}

The geriatric hip fracture occurs in a patient population subject to the bone weakening of osteoporosis. The incidence of such fracture increases with age after the sixth decade, because it is much more common in females (who live longer on the average), women with hip fractures outnumber their male counterparts by a margin of three to one. They also experience a higher percentage of the most common fracture associated with osteoporosis: the subcapital or femoral neck fracture. The femoral neck is the site of most severe involvement of osteoporosis within the hip joint. Fracture can occur just below the head of the femur, through the femoral neck (subcapital), and it can be caused by

the patient's sudden movement rather than by the fall which follows.

\section{Point of Concern: Coexisting Diseases}

The hip fracture patient population presents with significant coexisting disease, even considering their advanced age. More than half are classified ASA physical status III and a significant number are physical status IV. The most common reason for advanced physical status is heart disease, the majority with clinically evident coronary artery disease. Common in such patients are prior neurological problems (e.g., stroke, Parkinson's Disease and dementia) and the prevalence of multidrug therapy for coexisting disease. Side effects of polypharmacy, drug interactions, and electrolyte disturbances must be considered. The incidence of hip fracture in these patients may be increased by the presence of depression, dementia, confusion, and treatment with major tranquilizers, all of which may interact with potential anesthetic agents. Numerous agents (e.g., steroid therapy for rheumatoid arthritis) may accelerate bone weakening and increase the risk of hip fracture.

Hypoxemia is a constant factor in hip fracture patients; it may be related to coexisting disease, the pathophysiology of the fracture, or the effect of bed rest.. Pre- existing pulmonary compromise magnifies the pulmonary pathophysiology from the hip fracture (e.g., fat embolism or atelectasis). In advanced geriatric patients, lung function has decreased with age, and closing volume is very close to tidal volume. Some atelectasis occurs in the normal state and rapidly worsens with bed rest and pain from the 
hip fracture. Atelectasis is inevitable and increases the potential for pneumonia from defective secretion clearance.

Geriatric mortality is high during the initial admission for hip fracture (normally 10 percent during the admission and 20 percent within the first year). The most likely causes of death are pulmonary embolism (DVT related), pneumonia, cardiac failure, and acute myocardial infarction. All are incidence-related to age, prefracture physical status, and (inversely) ambulatory status. Conservative treatment of hip fracture (bedrest without surgery) accounts for a much higher mortality rate, owing to numerous complications (pneumonia, DVT, pulmonary embolism, or urinary tract sepsis).

\section{Psychological Factors}

The emotional significance of hip fracture to the geriatric patient must also be considered. Many cultures associate a fracture hip with the end of life, basing that belief on the high one-year mortality rate and the frequently decreased level of activity with survival. Aggressive education and frequent reassurance must be employed continuously to prevent this cultural norm from decreasing the patient's motivation and contributing to higher mortality. Agitation and disorientation can be caused by a high degree of anxiety combined with the effects of bed rest, pain, and the strange hospital environment. Family members can be significant in maintaining the orientation status of the acute hip fracture patient. It is wise to include them in the anesthesia preoperative visit. Having them understand clearly the plans to repair the hip, control pain, and proceed toward earliest possible ambulation will assist in maintaining orientation and mental status. It also provides a goal which increases motivation to survive.

\section{Anatomy}

There are three classes of geriatric hip fracture related to the site on the proximal femur where they occur. Their occurrence is related to the degree of osteoporosis at the site and the amount of trauma which has occurred. The subtrochanteric fracture is the most violent, is associated with the most blood loss, and is not related necessarily to osteoporosis. It causes the highest incidence of traumatic injuries to other organs, often occurring with motor vehiclerelated trauma: auto-passenger or autopedestrian accidents. The intertrochanteric fracture lies in the intermediate range of severity and occurs with significant trauma and moderate blood loss into the tissues. It can occur in osteoporotic hips with a violent mechanism of injury. The femoral neck fracture is most often associated with osteoporosis and may occur with relatively minor stress applied in just the right vector through very thin bone. Although presurgical blood loss is not trivial, it is the least of hip fractures. While the femoral neck fracture can be caused by a fall, many occur from violent movement and, in. turn, create the fall. Because of the smaller surface area of fracture and decreased cancellous bone, the blood loss is less.

The anatomical differences in hip fractures also dictate their treatment patterns. Subtrochanteric and intertrochanteric fractures occur in more viable bone, hence yield excellent prognosis for healing if there is anatomic internal fixation. There are wide fracture lines involved, rich with cancellous bone. The blood supply to the bone is usually not impaired. This condition dictates techniques which draw these fragments together and stabilize the reduction. In the femoral neck fracture, on the other hand, the fracture line occurs through the tissues which contain the blood supply to the femoral head. While the distal side remains well vascularized, the proximal side (including the entire femoral head and the proximal neck) is almost always deprived of blood supply, likely producing short-term avascular necrosis. The practical significance here is that hardware inserted to reduce the fracture will not necessarily cause healing, because one side of the fracture line will become avascular. With progressive weightbearing, the fixation devices tend to cut through the avascular bone. Any need for further surgery is caused by progressively increasing pain with attempted ambulation, either from the avascular necrosis or nonunion or impingement of the fixation devices on the bony joint surface of the acetabulum. The surgical strategy with these fractures focus on procedures for replacing the 
fractured femoral head and proximal neck with a prosthetic device and as with all hip fractures, encourage early ambulation.

\section{Surgical Considerations: Femoral Neck Fractures}

Because the probability of avascular necrosis of the femoral head is so high in this type of fracture, hemiarthroplasty is most commonly selected. The procedure may employ a simple ball and stem or the bipolar hemiarthroplasty involving a mobile head-neck (plastic on metal) interface within the prosthesis. The advantage of the bipolar prosthesis is the resultant mobile, prosthetic femoral head within the existing acetabulum, a feature that will afford less trauma to the acetabulum with similar ambulation and less damage to the acetabulum.

The patient is placed in the lateral or semi-lateral position. Incision and approach are similar to those for total hip replacement, as is the preparation of the femoral canal for the endoprosthesis. The progressive reaming of the canal is associated with steady blood loss, and the placement of the prosthesis can involve the use of methylmethacrylate if the native bone is very osteoporotic. The cementing can cause the same haemodynamic fluctuations that occur during other cemented joint replacements. These fluctuations are related to the vasodilatory and mast-cell degranulating properties of the monomeric form of methylmethacrylate; because these patients tend to be relatively sick with limited reserves, care must be taken to prepare the patient for cement use. Hypovolemia is poorly tolerated. Embolization of fat or air can result from pushing the stem of the prosthesis into the femoral canal (with or without the presence of cement), a condition that can further compromise the haemodynamic status of the patient if either ventilation or cardiac output are impaired by emboli.

The advantage of hemiarthroplasty lies in early rehabilitation of the patient and the resumption of a high degree of activity. However, the hemiarthroplasty can induce degenerative changes in the native acetabulum, which will become painful and, eventually, necessitate total hip replacement. At that point, the destruction of the acetabulum by the prosthetic femoral head could be severe enough to require more difficult procedures for acetabular arthroplasty. That possibility is eliminated when total hip replacement is chosen as the definitive treatment of acute hip fracture. This potential advantage must be considered in light of the disadvantages of longer surgical time, greater blood loss and haemodynamic change, and more painful, involved rehabilitation. More involved rehabilitation can defeat the primary purpose of the procedure (to achieve rapid ambulation) if the patient is not physically viable enough to rehabilitate the total hip arthroplasty.

The opposite extreme is the demented or extremely sick patient who sustains a femoral neck fracture. Here, total hip replacement is out of the question; because of morbidity, the patient is unlikely to be physically active enough to derive the benefit. Even the hemiarthroplasty may be an aggressive choice, due to the length of the surgical procedure and the haemodynamic changes associated with increased blood loss. In some cases, simple pinning of the fracture in the encountered position is chosen. This technique is selected for a patient expected to ambulate partially or minimally, but unable because of pain to tolerate the stress of conservative (nonsurgical) fracture management. The non ambulating patient faces increased morbidity from conservative hip fracture treatment because nursing care is so much more difficult and painful with the unstable fracture. Even simple internal fixation with limited probability of fracture union will decrease the pain from the site until fibrous union occurs. The pins can be placed percutaneously or by small incision with fluoroscopic assistance, either from the trochanter into the femoral head or via the medullary canal from a site as distal as the knee. Either involves minimal surgery and blood loss. The fracture thus becomes immobilized. Most importantly, fracture pain is relieved, and normal physiological functions of daily living can return to the pre-fracture state.

\section{Anesthetic Issues for Hip Fracture Patients}

The majority of preoperative anesthetic issues with geriatric hip fracture patients involve the amount of patient preparation for surgery in 
relation to the deleterious effects of painful bed rest during such preparation. The minimum preparation for this type of patient should start with the survey for other injuries from the trauma that precipitated the fracture. Any consideration of hip fracture surgery mandates this secondary trauma survey. The search must look for signs of intracranial injury; neck injury; injury to the thorax (especially pneumothorax from rib fracture, or more severe injuries such as myocardial and pulmonary contusion from fractures caused by greater trauma); intra-abdominal injuries (e.g., spleen rupture); and injuries to the pelvis or pelvic organs. Consideration must be given to the possibility that the fall which caused the fracture could have been precipitated by an acute cardiac or neurovascular incident.

\section{Preoperative Preparation}

Once the initial trauma assessment is complete, the interaction of coexisting diseases must be evaluated for potential impact on anaesthesia and surgery. At this point, it becomes relatively urgent to optimize the patient's condition for surgery. Although delay of 12 to 24 hours may be prudent for subspecialty consultation and intervention, it should be kept to the minimum necessary to achieve the best possible condition, always keeping in mind the deleterious effects of bed rest. The rate of mortality increases for surgery within 24 hours, and there is general agreement that it increases further as significantly more than 24 hours elapse.

Almost every geriatric hip fracture patient will present with hypoxemia at some point within the first 24 hours. The hypoxemia can be related to the influence of pain and bed rest on the decreased pulmonary reserves associated with aging. The high incidence of fat embolism with hip fracture repair and cemented endoprosthesis contributes further to pulmonary dysfunction. It can worsen this process if associated with rightsided heart dysfunction, a combination reported to lead to mortality from right-sided heart failure. When methylmethacrylate is used with hemiarthroplasty or total hip replacement, the probability of fat or marrow embolism may increase the potential of right-sided heart involvement.
The most common, serious coexisting disease encountered will be cardiac disease. Evaluation of the functional cardiovascular reserves may be difficult due to the bedridden state, the confusion encountered, and the fracture. Simple steps (e.g., auscultation, ECG, and chest x-ray) can detect acute decompensation. Echocardiography is feasible at the bedside and can give useful information about left ventricular and valvular function. Conventional stress testing is not possible, although alternative pharmacologic choices (dipyridamol-thallium scanning or dobutamine echocardiography) can be applied to detect the threshold for myocardial ischemia. If acute stroke is the precipitating cause of the fracture, evaluation of the patient's neurological condition may dictate the anaesthetic choice, because hemorrhagic stroke is a relative contraindication to regional anesthesia. Evaluation of electrolytes and blood count is required; anemia or electrolyte disturbances should be addressed prior to anesthesia induction. Even if blood loss will not be replaced with transfusion, the blood volume must be replaced with crystalloid or colloid to avoid precipitous drops in the blood pressure from anesthesia induction.

On arrival if the patients co morbidities or poor reserve does not allow immediate surgical intervention, we need to institute excellent quality of analgesia. The current advancements in regional anaesthetic techniques enables us to place perineural catheters eg continuous femoral nerve block for hip fracture. This site specific analgesia provides excellent quality of analgesia with least side effects. It not only helps to alleviate the pain and the discomfort but also gives a tremendous amount of positiveness and a sense of wellbeing for the patient.

The choice of anesthesia is determined by surgical factors, patient factors, and estimates of risk associated with anesthetic technique. For hip fracture repair, regional or general anesthesia can be selected. The current evidence favours a regional anaesthetic technique as there are certain proven advantages of regional techniques over general anaesthesia. The benefits include decreased intraoperative blood loss, early mobilization, decreased incidence of deep vein 
thrombosis, less postoperative nausea and vomiting and a generalized state of well being.

The regional anesthesia techniques commonly used are spinal or epidural anesthesia or a combined spinal epidural anaesthesia, psoas compartment with sciatic blocks can be used especially in patients with fixed cardiac output in whom a neuraxial block is not preferred due to possible haemodynamic changes specifically profound hypotension. The alternative option in fixed cardiac output states include segmental epidural, here the titrated doses of local anaesthetic administration and just blocking the segments involved offers the benefits of regional anaesthesia in critically ill patient and at the same time provides stable haemodynamics.

The choice between epidural and spinal anesthesia is partially determined by the surgeon's speed. In a teaching center, the learning of orthopedic residents may require the use of continuous techniques with an epidural or subarachnoid catheter rather than the one-shot spinal anesthetic. This option especially applies in those centers where the epidural catheter can be left in place and used for postoperative pain relief.

Technical factors also help to determine the choice between epidural and spinal block for hip fracture. Epidural techniques require more time for placement and onset, with acute fracture positioned in a painful manner for placement of the block; the patient may be more able to tolerate the quick spinal block. The dose of local anesthetic is much higher for epidural anesthesia, decreasing the safety margin compared to spinal anesthesia. Conversely, the abrupt onset of the spinal anesthetic may be poorly tolerated in the hypovolemic or anemic patient with limited cardiac reserves, thus the epidural may be preferable because local anesthetic dose can be administered in increments. While the continuous spinal technique offers the same advantages of titrated dosing and low rates of spinal headache, the risk of meningitis dampens any enthusiasm for keeping these catheters for postoperative pain control.
The combined spinal epidural anaesthesia offers the advantage of both. A lower dose of local anaesthetic is administered intrathecally so that there are no haemodynamic changes, the only important factor is to precisely check the level of surgical anaesthesia after a low dose and if any segments are spared we could give epidural supplements so as to achieve the desired level of surgical anaesthesia.

\section{Outcome}

In the anesthetic literature, numerous comparisons have been made between regional and general anesthesia for hip fracture surgery. They progressed to a point where the lay press reported better outcomes after hip fracture when the surgery was performed with spinal anesthesia. As always, the truth is not as clear. Several issues have clear outcomes, other outcomes and overall outcome studies are controversial.

There is a clear advantage to regional anesthesia for hip fracture surgery with a decrease in intraoperative blood loss and postoperative deep venous thrombosis (DVT). Blood loss and the amount of transfusion will be consistently less with regional anesthesia. The cause of this favorable effect is probably a combination of lower average blood pressure when blood loss is occurring and the dilation of capacitance vessels deep to the hip wound, resulting in dependent drainage of blood away from the operative site. Decreased blood loss may decrease the potential for intraoperative haemodynamic changes.

Pulmonary embolism and DVT are significantly less in hip fracture patients having regional anesthesia than in those having general anesthesia. Since pulmonary embolism is one of the primary causes of mortality after hip fracture, this alone should be an indication for the use of regional anesthesia. The majority of deep venous clots probably form intraoperatively, caused by combining vessel trauma from stretch with venous pooling. Spinal or epidural anesthesia might slightly influence vessel trauma by intense relaxation; the more likely etiology of decreased thrombus formation is the increased flow to the tissues during a regional anesthetic, when the blood pressure is 
maintained within reason- able limits. Postoperative DVT prophylaxis and detection efforts are still necessary.

There may be a difference in mental status and alertness related to anesthetic technique. Hip fracture is known to be associated with preoperative confusion and hypoxemia. Platelet factors may contribute to hypoxemia and subclinical fat embolism.It is generally agreed that the oxygenation of the patient in the immediate postoperative period will be significantly higher after regional anesthesia than during recovery from general anesthesia. As many as one-third will remain con- fused for 24 hours, significantly less when spinal anesthesia is compared to general anesthesia. This differentiation is probably caused by residual effects of potent inhalation agents and anticholinergics. Prior psychiatric illness increases this risk and the incidence is especially high in patients with preexisting mild dementia or depression. Regardless of the etiology, confused patients have a significantly increased length of stay in the hospita1. Studies are not as conclusive about the variations in continuing confusion caused by regional and general anesthesia, hence it is possible that there is no significant difference. Spinal anesthesia may decrease the perioperative incidence of pneumonia compared to general anesthesia.

The decrease in DVT and confusion in the shortterm postoperative period may also be related to the fact that regional anesthesia decreases the interval between solid hip repair and first ambulation attempts. A considerable heat loss in the perioperative period of hip fracture repair results from the large amount of body surface area exposed for the surgery. There is no apparent difference between spinal and general anesthesia as regards the amount of hypothermia produced.

Overall outcome after hip fracture surgery reflects an 8 to 10 percent mortality within eight months of the surgery. The influence of anesthetic technique on out- come variables has shown a preference for regional anesthesia rather than general anesthesia, relating to the DVT rate, blood loss, decreased stress response (ADH, epinephrine, norepinephrine), and the incidence of profound dementia. However, large series comparing similar patients at similar time intervals from fracture documented no difference between groups for mortality. Non surgical treatment is associated with at least double the mortality.

\section{Techniques for Regional Anesthesia for Hip Fracture Repair}

There is a challenge in the conduct of spinal or epidural anesthesia in the patient with acute hip fracture. The patient is in bed in the supine position, so that the slightest movement can cause severe pain. In intertrochanteric fractures, the fracture fragments present a risk to the sciatic nerve if they are manipulated in an uncontrolled manner. Positioning the patient into the lateral position may require intravenous administration of narcotic analgesics and the presence of a member of the surgical team. Pain will make it difficult to achieve the spinal flexion normally sought to facilitate dural puncture. It can be partially accomplished by flexing the upper body and the unaffected hip. In most cases, the fractured hip is turned to the superior side and held by the orthopedic surgery team in the least painful manner. Preoperative femoral block could be routinely performed to all these patients who help to position the patients comfortably for placing a neuraxial block.

The spinal anesthetic equipment should be prepared prior to positioning. Once the patient is in place, the block should be achieved as rapidly as possible. If epidural anesthesia is chosen, injection of local anesthetic should occur as fast as safely possible. With spinal anesthesia, neartotal relief of the acute fracture pain occurs within moments of the local anesthetic injection, allowing (with slight hesitation) a pain-free turn back to supine and into the hip surgery position. It is also frequently noted that patients immediately relax and often spontaneously sleep for the first time since the fracture. The combination of this reaction and their advanced age makes it mandatory to avoid ventilatory embarrassment by very conservative use of further intravenous sedative drugs.

General anesthesia can be a technical challenge if minimizing pain necessitates induction on the 
orthopedic bed and transfer under general anesthesia to the operating table. Access to the airway can also be challenging. To avoid injury from unstable fracture fragments in osteoporotic extremities or structures in the ipsilateral lower extremity, care must be taken with transfer of the anesthetized patient to a fracture table or into the lateral position. A further challenge is presented in deter mining the emergence of confused or demented geriatric patients from anesthesia.

Immediate postoperative care should be directed to supporting oxygenation, controlling pain, and facilitating the patient's return to the baseline mental status by emphasizing orientation. Appropriate pain control should be established prior to release from the recovery room, to set a safe template for further analgesia on the regular nursing floor. Poor pain control may contribute to increased confusion in advanced geriatric hip fracture patients. Postoperative pain therapy is best a multimodal approach. A continuous infusion of $0.125 \%$ or $0.0625 \%$ Bupivacaine with 2-3microgram Fentanyl/ $\mathrm{ml}$ at a rate of 6 to $8 \mathrm{ml} /$ hour either through the epidural or through the continuous perineural catheter offers an excellent quality of analgesia. In the multimodal therapy the local anaesthetic infusions can be supplemented with analgesics including a combination of paracetamol, tramadol, NSAID(when there is no contraindication) and opioids. The VAS scores have to be ideally below 3 .

The trigger haemoglobin level at which a transfusion has to be adminiatered would be $10 \mathrm{gms} \%$ in the presence of cardiorespiratory compromise and $8 \mathrm{gms} \%$ in the others. Hyponatraemia is a common electrolyte abnormality that is noticed in these patients. Hence we need to closely monitor Serum sodium levels in the postoperative period. Adequate hydration and oral intake have to be monitored as dehydration is very common in these patients who suddenly decrease their oral intake due to mood swings. Most of these patients suffer from constipation and hence stool softners could be made a part of their postoperative drug regimen.

\section{Conclusion:}

Geriatric patients with hip fracture offer a great challenge to the anaesthesiologists. A careful preoperative examination, preoperative optimization, safe intraoperative anaesthetic techniques, good postoperative pain relief, good postoperative follow up with rehabilitation would aid in decreasing the morbidity in these patients.

\section{References}

1. Anaesthesia for Orthopaedic surgery by Andre P. Boezaart.

2. Anaesthesia for High Risk Patient by Ian McConachie. 